\title{
3D Markov chain based multi-priority path selection in the heterogeneous Internet of Things
}

\author{
Huan $\mathrm{Wu}^{1,2}$, Xiangming $\mathrm{Wen}^{1}$, Zhaoming $\mathrm{Lu}^{1}$ and $\mathrm{Yao} \mathrm{Nie}^{2 *}$ \\ ${ }^{1}$ Beijing Laboratory of Advanced Information Networks, Beijing Key Laboratory of Network System Architecture \\ and Convergence, Beijing University of Posts and Telecommunications \\ Beijing, 100876 - China \\ ${ }^{2}$ School of Electronics and Information Engineering, West Anhui University, Lu'an, 237012 - China \\ [e-mail: wuhuan279@gmail.com, xiangmw@bupt.edu.cn, lzy_0372@163.com,buptnieyao@gmail.com] \\ *Corresponding author: Yao Nie
}

Received December 12, 2018; revised April 9, 2019; accepted June 18, 2019; published November 30, 2019

\begin{abstract}
Internet of Things (IoT) based sensor networks have gained unprecedented popularity in recent years. With the exponential explosion of the objects (sensors and mobiles), the bandwidth and the speed of data transmission are dwarfed by the anticipated emergence of IoT. In this paper, we propose a novel heterogeneous IoT model integrated the power line communication (PLC) and WiFi network to increase the network capacity and cope with the rapid growth of the objects. We firstly propose the mean transmission delay calculation algorithm based the 3D Markov chain according to the multi-priority of the objects. Then, the attractor selection algorithm, which is based on the adaptive behavior of the biological system, is exploited. The combined the 3D Markov chain and the attractor selection model, named MASM, can select the optimal path adaptively in the heterogeneous IoT according to the environment. Furthermore, we verify that the MASM improves the transmission efficiency and reduce the transmission delay effectively. The simulation results show that the MASM is stable to changes in the environment and more applicable for the heterogeneous IoT, compared with the other algorithms.
\end{abstract}

Keywords: PLC, heterogeneous IoT, path selection, Markov chain, attractor selection

This work is supported by the National Natural Science Foundation of China (61671073), the WLAN Achievement Transformation based on Software-Defined Networking (SDN) of Beijing Municipal Commission of Education (201501001), the project of Young Core Teacher in Higher Education of Anhui (gxyq2019070) and the Scientific Research Foundation of the Higher Education Institutions of Anhui Province (KJ2019A0630). 


\section{Introduction}

Di fferent from the traditional networks which take human being as the center, Internet of Thing (IoT) takes the objects (sensors, devices) as the core and connects every object to the Internet, will identify and communicate with other objects. Currently, IoT technology is used in many important fields such as smart grid, smart home, city management, urban intelligent transportation, health care, precision agriculture, ecological environment and resources monitoring [1], [2]. Those applications of the "intelligence" provided by IoT are based on the large-scale interconnection of the objects. However, the IoT smart objects are expected to reach 212 billion entities deployed globally at the end of 2020, in which the machine-to-machine (M2M) traffic flows are expected to constitute up to $45 \%$ of the whole Internet traffic [3]. Moreover, the World Wireless Research Forum predicts that there will be the ubiquitous IoT world with more than 7 trillion sensors providing ubiquitous services to the users in 2030 [4]. Due to connecting the billions of objects with the various performances, the IoT needs the compatible network architecture urgently. In additional, more sufficient bandwidth and ubiquitous network coverage are considered in IoT. Therefore, building the compatible IoT architecture to increase the capacity, connect the objects seamlessly and allocate the appropriate network resources dynamically are the inevitable problems in IoT.

In the traditional heterogeneous networks, the researchers apply the Ultra Dense Networks (UDN) to increase the network capacity [5]. That is to say, more base stations are deployed in the networks which are a form of the super-dense heterogeneous network. By far, the main structures of the heterogeneous wireless networks are WLAN - worldwide interoperability for microwave access (WiMax), WiMax - WiFi, WLAN - universal mobile telecommunications system (UMTS), WiMax - 3G (3rd generation), etc.[6]-[8]. Simultaneously, the spectral efficiency of the wireless communication paths approximates to Shannon limit based the traditional orthogonal frequency division multiplexing (OFDM) and the multiple input multiple output (MIMO). However, these structures cannot completely solve the problem of the network capacity due to the wireless spectrum resources limited, while the network capacity requirements grow with the number of the objects increased. In additional, to provide the more coverage requires more base stations deployed, and more construction and maintenance costs. Fortunately, the power line communication (PLC) is now a novel kind of the high speed information transmission technology over the power line, which is mainly used in the "last mile" of the user access and the intranet. Because power line is the commonest and widest coverage physical media, the operators apply the power line to transmit data and the exchange information for reducing the costs and the new communication networks construction. Thus, the PLC has become a hotspot in the development of information transmission technology as a supplement to the wireless networks. Power line has obvious advantages in the wider coverage areas, stability and reliability, which are important and cost-effective for users in IoT to coverage the blind areas and hotspots, such as the countryside, mountain area, no-human-zone and commercial hotspots or community hotspots. In our previous work [9], [10], the PLC has been used as a transmission medium to integrate the wireless network (WN) to achieve better capacity, low-cost performances, etc.

One of the key tasks in the heterogeneous network is how to select the path (route) between the source and the destination for the effective data transmission [11], [12]. There are two classifications of the path selection algorithms, the path selection decision criteria and the path decision selection algorithm. On the one hand, the path selection decision criteria can be 
divided into the single-criterion and the multi-criteria according to the applications and the user requirements. The common path decision single-criterion is based on the received signal strength (RSS), which is important for the mobile equipment to keep seamless connection [13]-[15]. The 3GPP has proposed the biased access control based on RSS in release 10, which guarantees the loading balance of the network [16]. To better satisfy the requirement of the upper-layer applicaions in the heterogeneous netwroks, a handover algorithm considers the available bandwidth as the link-layer parameters for the path selection [17]. In this algorithm, a moble node moves out or keeps on its present network according to the threshold of the bandwith. [18] has proposed a network condition detection algorithm to estimate the available bandwidth and the packet delay while the calculated result is larger than the predescribed threshold. The network load is also one of the single-criterion to be considered. In [19], the author has developed a vertical handoff decision algorithm which balances the network load among all the attachment points (e.g, the base stations and the access points). Moreover, some researchers focused on the multi-criteria path selection decision to consider the variety of constraints. In [20], a united decision criterion has approached, which includes the network metrics in terms of device relation, application requirements and user preferences. [21] has proposed a novel fuzzy-logic (FL)-based decision-making algorithm based on the network handoff cost, delay and available bandwidth as the network selection decision criteria. On the other hand, the network decision selection algorithm is proposed further based on the network selection criteria. One of the most widely used algorithm was multiple attribute decision making (MADM), which has four steps, the including selection of the decision criteria, the collection of values for the selected criteria, the criteria weights and the ranking of the alternatives [22]. Based on the MADM, [23] proposed a Markov decision process of the network decision selection algorithm which decreases the long handoff delays and connection latency. Since the decision criteria have a fixed threshold, the path selection works while the threshold conditions is triggered, rather than selecting the network adaptively. In conclusion, the path selection in the current works are limited by the decision criteria.

In this paper, a novel heterogeneous IoT model integrated the PLC and WN is proposed, which combines the PLC and WN to develop the bandwidth and coverage, and increase the stability and robustness. To transmit data seamlessly, firstly we apply an effective virtual layer (EVL) to convert the protocols and formats of data packet (frame). Then, the adaptive network selection algorithm based attractor selection algorithm combining the 3D Markov chain (MASM) is proposed to provide the better allocation of the network resources. Considering data (flow) priority, the 3D Markov chain calculates different queues length of data according to the different priorities. Moreover, the MASM can select an optimal path adaptively according to the external environment (the transmission delay of different priorities). The simulation results show that the MASM can select the optimal path dynamically compared with the Ad hoc On-demand Distance Vector Routing (AODV), greedy algorithm (GA), Simple Additive Weighting (SAW) and Interference-Suppression Resource Optimization based on Potential Game (ISRO_PG). Especially, in the scenaio with a large number of nodes, the delivery ratio of the MASM is better than other algorithms.

The rest of this paper is organized as follow. System model is surveyed in section 2. We show the mathematical model of the queuing and the attractor selection algorithm in Section 3. This is followed by a description of how to apply the attractor selection in the heterogeneous IoT in Section 4 and then we evaluate its performance by some exemplary simulation results which are presented in section 5. Finally, this paper is concluded in Section 6. 


\section{System Model}

In this section, we propose the system model of the heterogeneous IoT. Firstly, the virtual layer is defined for the protocols and formats conversion to hide the details of the different access technologies. Then, we provide the queuing model, which is the foundation of the mathematical calculation for the queue length. Finally, we discuss the control model which can control the whole path selection process.

\subsection{Network Model}

In the heterogeneous IoT, we assume that the PLC and WiFi coverage always exist. Each object (device) and transmission node accesses to the PLC and WiFi through the access point (AP) or PLC access point (PAP), respectively. Fig. 1 shows the scenario that the objects (devices) access to the heterogeneous IoT. Every node gets the path information according to the path feedback, and transmits and receives data in a rate depending on the user's mobility (e.g. immobilization, pedestrian, vehicular) and the environment (e.g. rural, urban, mountain area). Fig. 2 shows the simple access scenario model. Then, we detailed introduce the Effective Virtual Layer (EVL), the queuing model and the control model of the heterogeneous IoT network model.

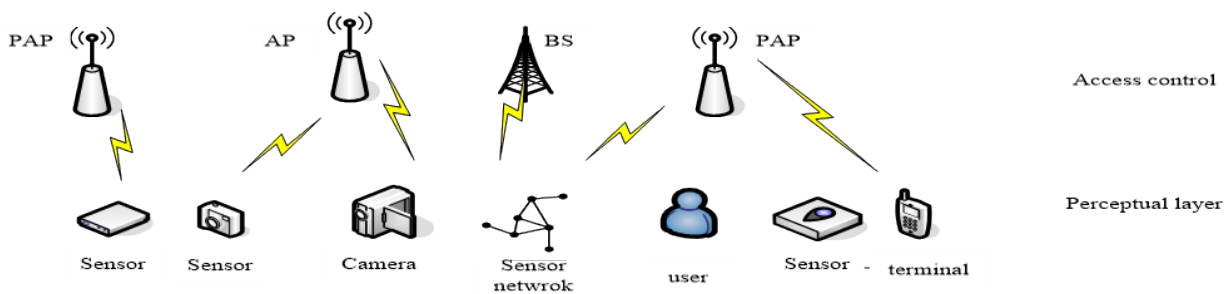

Fig. 1. Access model of the heterogeneous IoT

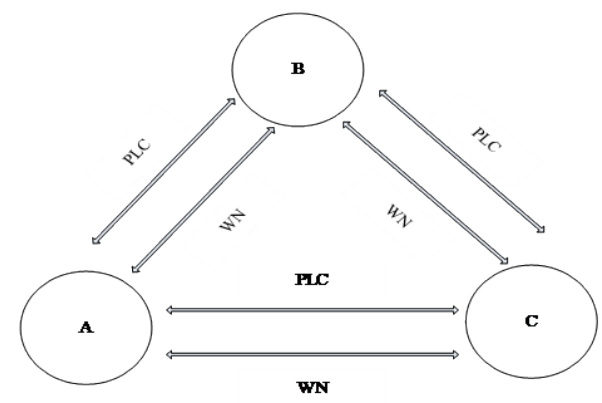

Fig. 2. The simple access model

\subsection{Effective Virtual Layer}

In the IoT network, the protocols and formats of the packet are different for the different access technologies, such as the limited packet size and the various address lengths [24], [25]. Therefore, we assume the EVL, which is an abstraction layer, has capable of harmonizing the access with a common language and procedure for the different access technologies. The EVL is located between the technological and the application levels as shown in Fig. 3. Its feature of hiding the details of different access technologies is fundamental to exempt the upper application from issues. Thus, the EVL consists of two main sub-layers: the interface and the communication sub-layers. The interface sub-layer provides a compatible interface which allows the various technologies to access to the heterogeneous IoT. It is responsible for the 
management of all the incoming / outcoming messaging operations involved in the communication with the external environment. And the communication sub-layer is a conversion layer, which makes the protocols and the formats of the packet conversion into a standard service language. As shown in Fig. 3, the header of the packet is added and resealed in the EVL, which hides the diversity of transmission media without changing the existing path. Based on the conversion process, we assume that the objects can access to the heterogeneous IoT through the PLC or the WN with ubiquitous and seamless connectivity.

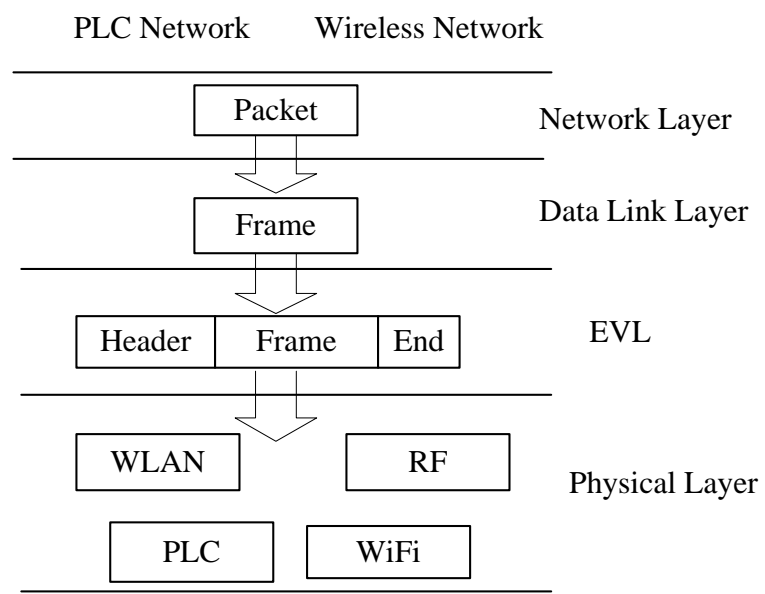

Fig. 3. The wrapping process in the EVL

\subsection{Queuing Model}

The explosive growth of data of the IoT application can be divided into different types, the inquiry data, the control data and the monitoring data. The different types of data have the different requirements for Quality of Service (QoS). Considering the requirements of QoS of the heterogeneous IoT, we divide the different types of data into the two classifications including the high priority and the low priority. When data (e.g. file, packet) arrives the node to find another data currently being queued or in transmission, the data stands in a queue. Based on the previously stated assumptions, our system can be modeled with server vacations and impatient customers, or reneging (their deadline expires) [26]-[28]. There are two queue states corresponding to the PLC states and the WiFi states. Each queue state has two priorities corresponding to the high priority and the low priority. The high priority data requires the less delay and low deadline, such as the real time monitoring, the streaming video and voice. Correspondingly, the low priority data requires the high tolerance delay and high deadline, such as no real time monitoring, data acquisition. If the deadline for data expires (waiting in the queue), the data abandons the current network and is transmitted through the other network based the MASM, which will be explained in detail later. We also assume that the queuing obeys the First Come First Served (FCFS) queuing discipline, and the queuing model is in steady state as shown in Fig. 4.

\subsection{Control Model}

The control model consists of four parts, the feedback module, the algorithm module, the storage module and the scheduling module. The feedback module collects the path information (congestion, disconnection, etc.) between the two nodes which communicate with each other. The information is transmitted to the algorithm module to provide an environmental basis for the path selection. Here, the algorithm module is used to select the 
optimal path, which is the core in the control model. The storage module is used to store the important information, such as the result of MASM. Moreover, the scheduling module is mainly used for the path adjustment and switching process. While the object's data is coming into the node, the scheduling modular puts them into a suitable queue of the path according to the result of MASM.

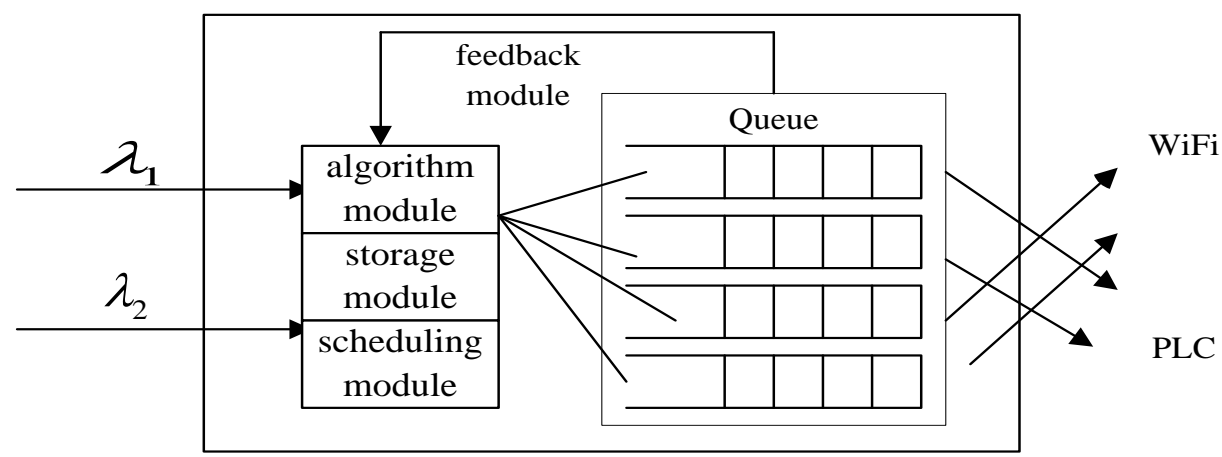

Fig. 4. The queuing model in the heterogeneous IoT

\section{Mathematical for System Model}

In this section, the problem of different services queuing is solved by 3D Markov chain. According to [28], [29] and considering the characteristic of the PLC, Theorem 1 and Theorem 2 are proved. These two theorems represent the mean transmission delay of the different services which impact the basic attractor selection model (ASM) for the path selection. Then, we extend the ASM and define the attractors related to the mean transmission delay.

\subsection{The Mean Transmission Delay Calculation}

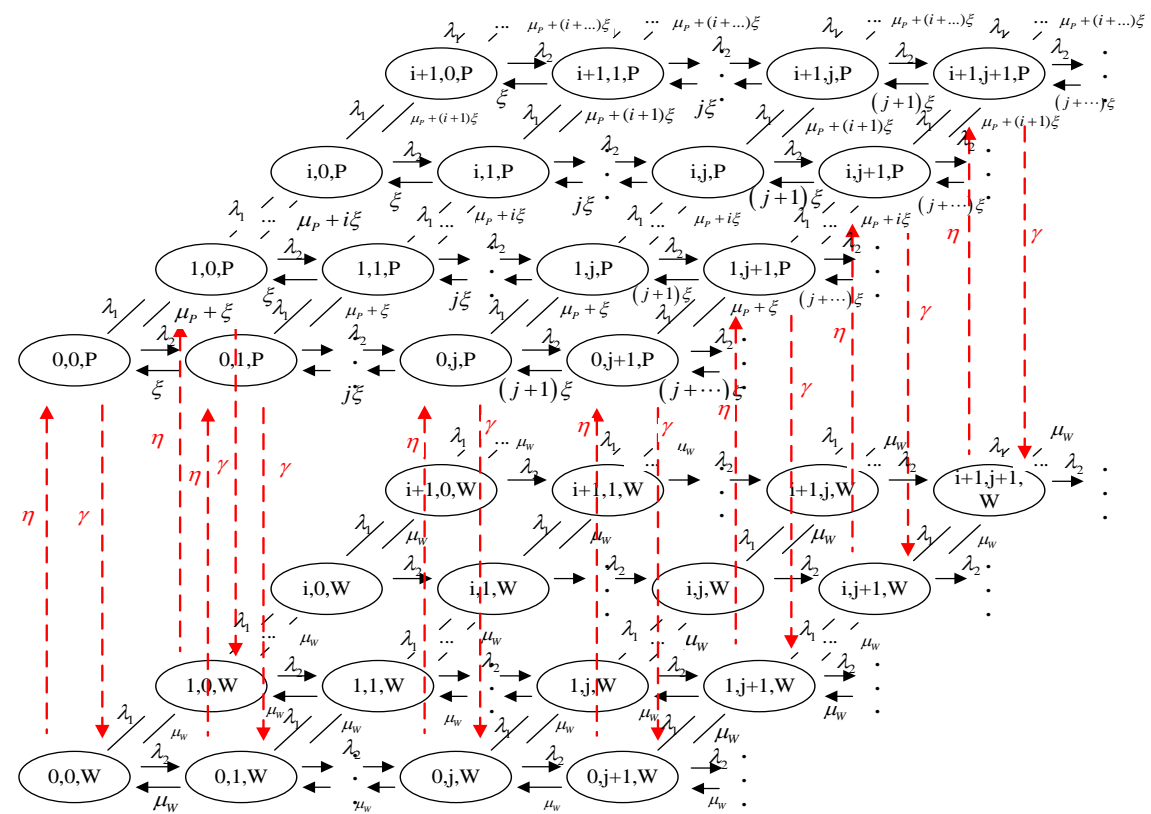

Fig. 5. 3D Markov chain 
Let queuing phenomenon be treated as a $M / M / 1$ type queue operated in the 3D Markov chain as shown in Fig. 5. It is assumed that the Markov process is independent of the arrival, service and impatience processes. Let $\pi_{P}$ and $\pi_{C}$ be the steady-state probabilities of the queuing environment of the PLC and WiFi, respectively. $i$ and $j$ denote the low priority data and the high priority data in the 3D Markov chain, respectively. The states of the WiFi connectivity are denoted as $\{i, j, W\}$, and the states of the PLC connectivity are denoted as $\{i, j, P\}$. During the PLC states and the WiFi states, the arrival rates are $\lambda_{1}$ and $\lambda_{2}$, which are correspond to the low and high priority, respectively. And during the PLC states, the service rates are $u_{P}+i \xi$ and $j \xi$, which are correspond to the low priority and high priority, respectively. During the WiFi states, the service rate is $u_{W}$. Then, Theorem 1 and Theorem 2, which are the mathematical expressions of the mean transmission delay of the different priorities, are given in the next paragraph. The useful notations are depicted in Table 1.

Table 1. Variables and Notation

\begin{tabular}{|c|l|}
\hline \multicolumn{1}{|c|}{ Variable } & \multicolumn{1}{c|}{ Definition } \\
\hline$\lambda_{1}$ and $\lambda_{2}$ & The arrival rates of the low and high priority data, respectively \\
\hline$\pi_{i, j . P}$ & $\begin{array}{l}\text { Stationary probability of finding the ith low and } j \text { th high priority data in the } \\
\text { PLC state }\end{array}$ \\
\hline$\pi_{i, j, W}$ & $\begin{array}{l}\text { Stationary probability of finding the ith low and the } j \text { th high priority data in } \\
\text { the WiFi state }\end{array}$ \\
\hline$\pi_{P}$ & Probability of finding the state under the PLC only \\
\hline$\pi_{W}$ & Probability of finding the state under the WiFi only \\
\hline$\eta$ & The rate of leaving the WiFi state \\
\hline$\gamma$ & The rate of leaving the PLC state \\
\hline$\mu_{W}$ & The service rate while in the WiFi state \\
\hline
\end{tabular}

Theorem 1. The mean transmission delay of the low priority data is

$$
\begin{aligned}
& E\left(T_{1}\right)=\frac{1}{\lambda_{1}}\left[\frac{\lambda_{1}-\mu_{W}\left[\pi_{W}-F_{0}^{W}(1)\right]-\mu_{P}\left[\pi_{P}-F_{0}^{P}(1)\right]}{\eta}\right] \\
& +\frac{1}{\lambda_{1}}\left[\frac{\lambda_{1}\left(\eta \pi_{W}+\gamma\right)-(\gamma+\eta) \mu_{W}\left[\pi_{W}-F_{0}^{W}(1)\right]-\gamma \mu_{P}\left(\pi_{P}-F_{0}^{P}(1)\right)}{\eta^{2}}\right]
\end{aligned}
$$

Proof: we use probability generating functions (PGF) to derive the mean transmission delay of this queue, which is known for a long time in the 2D Markov chains [29], [30]. Writing the balance equations for the 3D Markov chain in the PLC coverage, we have

$$
\begin{gathered}
\left(\lambda_{1}+\lambda_{2}+\gamma\right) \pi_{0,0, P}=\left(\mu_{P}+\xi\right) \pi_{1,0, P}+\xi \pi_{0,1, P}+\eta \pi_{0,0, W} \\
\left(\lambda_{1}+\lambda_{2}+j \xi+\gamma\right) \pi_{0, j, p}=\left(\mu_{P}+\xi\right) \pi_{1, j, P}+(j+1) \xi \pi_{0, j+1, P}+\lambda_{2} \pi_{0, j-1, P}+\eta \pi_{0, j, W}(j \geq 1) \\
{\left[\lambda_{1}+\lambda_{2}+\left(\mu_{P}+i \xi\right)+\gamma\right] \pi_{i, 0, P}} \\
=\left[\mu_{P}+(i+1) \xi\right] \pi_{i+1,0, P}+\xi \pi_{i, 1, P}+\lambda_{1} \pi_{i-1,0, P}+\eta \pi_{i, 0, W}(i \geq 1) \\
{\left[\lambda_{1}+\lambda_{2}+\left(\mu_{P}+i \xi\right)+j \xi+\gamma\right] \pi_{i, j, p}=\left[\mu_{P}+(i+1) \xi\right] \pi_{i+1, j, P}} \\
+(j+1) \xi \pi_{i, j+1, P}+\lambda_{1} \pi_{i-1, j, P}+\lambda_{2} \pi_{i, j-1, P}+\eta \pi_{i, j, W}(i \geq 1, j \geq 1)
\end{gathered}
$$


(2), (3), (4) and (5) are considered as the representations of a law relating to the steady state regime. The average rate at which a point (i.e. a state) is equals to the average rate at which a transition from the point occurs [29]. Similarly, in the WiFi coverage, we have

$$
\begin{gathered}
\left(\lambda_{1}+\lambda_{2}+\eta\right) \pi_{0,0, W}=\mu_{W} \pi_{1,0, W}+\mu_{W} \pi_{0,1, W}+\gamma \pi_{0,0, P} \\
\left(\lambda_{1}+\lambda_{2}+\mu_{W}+\eta\right) \pi_{0, j, W}=\mu_{W} \pi_{1, j, W}+\mu_{W} \pi_{0, j+1, W}+\lambda_{2} \pi_{0, j-1, W}+\gamma \pi_{0, j, P}(j \geq 1) \\
\left(\lambda_{1}+\lambda_{2}+\mu_{W}+\eta\right) \pi_{i, 0, W}=\mu_{W} \pi_{i+1,0, W}+\lambda_{1} \pi_{i-1,0, W}+\gamma \pi_{i, 0, P}(i \geq 1) \\
\left(\lambda_{1}+\lambda_{2}+\mu_{W}+\eta\right) \pi_{i, j, W}=\mu_{W} \pi_{i+1, j, W}+\lambda_{1} \pi_{i-1, j, W}+\lambda_{2} \pi_{i, j-1, W}+\gamma \pi_{i, j, P}(i \geq 1, j \geq 1)
\end{gathered}
$$

We define the joint PGFs of the PLC and the WiFi states as $F_{P}\left(z_{1}, z_{2}\right)=\sum_{i=0}^{\infty} \sum_{j=0}^{\infty} \pi_{i, j, P} z_{1}^{i} z_{2}^{j}$, $\left(\left|z_{1} \leq 1\right|, \mid z_{2} \leq 1\right)$ and $F_{W}\left(z_{1}, z_{2}\right)=\sum_{i=0}^{\infty} \sum_{j=0}^{\infty} \pi_{i, j, W} z_{1}^{i} z_{2}^{j},\left(\left|z_{1} \leq 1\right|,\left|z_{2} \leq 1\right|\right)$, respectively. And set $F_{i}^{P}\left(z_{2}\right)=\sum_{j=0}^{\infty} \pi_{i, j, P} z_{2}^{j}, F_{i}^{W}\left(z_{2}\right)=\sum_{j=0}^{\infty} \pi_{i, j, W} z_{2}^{j}$, then multiply (3), (4) and (5) with $z_{2}^{j}, z_{1}^{i}, z_{1}^{i} z_{2}^{j}$, respectively. Adding these equations together into (2), then we obtain

$$
\begin{aligned}
& \left(\lambda_{1}+\lambda_{2}+\gamma\right) F_{P}\left(z_{1}, z_{2}\right)+\mu_{P}\left[F_{P}\left(z_{1}, z_{2}\right)-F_{0}^{P}\left(z_{2}\right)\right]+\xi z_{1} \frac{\partial F_{P}\left(z_{1}, z_{2}\right)}{\partial z_{1}} \\
& +\xi z_{2} \frac{\partial F_{P}\left(z_{1}, z_{2}\right)}{\partial z_{2}}=\frac{\mu_{P}}{z_{1}}\left[F_{P}\left(z_{1}, z_{2}\right)-F_{0}^{P}\left(z_{2}\right)\right]+\xi \frac{\partial F_{P}\left(z_{1}, z_{2}\right)}{\partial z_{2}} \\
& +\xi \frac{\partial F_{P}\left(z_{1}, z_{2}\right)}{\partial z_{1}}+\lambda_{2} z_{2} F_{P}\left(z_{1}, z_{2}\right)+\lambda_{1} z_{1} F_{P}\left(z_{1}, z_{2}\right)+\eta F_{W}\left(z_{1}, z_{2}\right)
\end{aligned}
$$

Applying the same methods for (6) - (9), we get

$$
\begin{aligned}
& \left(\lambda_{1}+\lambda_{2}+\eta\right) F_{W}\left(z_{1}, z_{2}\right)+\mu_{W}\left[F_{W}\left(z_{1}, z_{2}\right)-\pi_{0,0, W}\right] \\
= & \frac{\mu_{W}}{z_{1}}\left[F_{W}\left(z_{1}, z_{2}\right)-F_{0}^{W}\left(z_{2}\right)\right]+\frac{\mu_{W}}{z_{2}}\left[F_{0}^{W}\left(z_{2}\right)-\pi_{0,0, W}\right] \\
& +z_{1} \lambda_{1} F_{W}\left(z_{1}, z_{2}\right)+z_{2} \lambda_{2} F_{W}\left(z_{1}, z_{2}\right)+\gamma F_{P}\left(z_{1}, z_{2}\right)
\end{aligned}
$$

Using appropriate mathematical transformation to (10) and (11), we get

and

$$
\begin{aligned}
& \lambda_{1}\left(1-z_{1}\right) F_{P}\left(z_{1}, z_{2}\right)+\lambda_{2}\left(1-z_{2}\right) F_{P}\left(z_{1}, z_{2}\right)+\gamma F_{P}\left(z_{1}, z_{2}\right)+\mu_{P}\left(1-\frac{1}{z_{1}}\right)\left[F_{P}\left(z_{1}, z_{2}\right)\right] \\
& +\xi\left(z_{1}-1\right) \frac{\partial F_{P}\left(z_{1}, z_{2}\right)}{\partial z_{1}}+\xi\left(z_{2}-1\right) \frac{\partial F_{P}\left(z_{1}, z_{2}\right)}{\partial z_{2}}=\eta F_{W}\left(z_{1}, z_{2}\right)+\mu_{P}\left(1-\frac{1}{z_{1}}\right) F_{0}^{P}\left(z_{2}\right)
\end{aligned}
$$

$$
\begin{aligned}
& \lambda_{1} z_{1} F_{W}\left(z_{1}, z_{2}\right)+\lambda_{2} z_{2} F_{W}\left(z_{1}, z_{2}\right)+\gamma F_{P}\left(z_{1}, z_{2}\right)=\left(\lambda_{1}+\lambda_{2}+\eta\right) F_{W}\left(z_{1}, z_{2}\right) \\
& +\mu_{W}\left(1-\frac{1}{z_{1}}\right)\left[F_{W}\left(z_{1}, z_{2}\right)-F_{0}^{W}\left(z_{2}\right)\right]+\mu_{W}\left(1-\frac{1}{z_{2}}\right)\left[F_{0}^{W}\left(z_{2}\right)-\pi_{0,0, W}\right]
\end{aligned}
$$

Take $z_{2}=1$ into (12) and (13), then (12) and (13) can be rewritten as 


$$
\begin{aligned}
& \lambda_{1}\left(1-z_{1}\right) F_{P}\left(z_{1}, 1\right)+\gamma F_{P}\left(z_{1}, 1\right)+\mu_{P}\left(1-\frac{1}{z_{1}}\right)\left[F_{P}\left(z_{1}, 1\right)\right]+\xi\left(z_{1}-1\right) \frac{\partial F_{P}\left(z_{1}, 1\right)}{\partial z_{1}} \\
& =\eta F_{W}\left(z_{1}, 1\right)+\mu_{P}\left(1-\frac{1}{z_{1}}\right) F_{0}^{P}(1)
\end{aligned}
$$

and

$$
\lambda_{1} z_{1} F_{W}\left(z_{1}, 1\right)+\gamma F_{P}\left(z_{1}, 1\right)=\left(\lambda_{1}+\eta\right) F_{W}\left(z_{1}, 1\right)+\mu_{W}\left(1-\frac{1}{z_{1}}\right)\left[F_{W}\left(z_{1}, 1\right)-F_{0}^{W}(1)\right]
$$

Furthermore, (14) and (15) yield, respectively

$$
\xi\left(1-z_{1}\right) \frac{\partial F_{P}\left(z_{1}, 1\right)}{\partial z_{1}}=\left[\lambda_{1}\left(1-z_{1}\right)+\gamma+\mu_{P}\left(1-\frac{1}{z_{1}}\right)\right] F_{P}\left(z_{1}, 1\right)-\mu_{P}\left(1-\frac{1}{z_{1}}\right) F_{0}^{P}(1)-\eta F_{W}\left(z_{1}, 1\right)
$$

and

$$
\left[\left(\lambda_{1} z_{1}-\mu_{W}\right)\left(1-z_{1}\right)+\eta z_{1}\right] F_{W}\left(z_{1}, 1\right)=\gamma z_{1} F_{P}\left(z_{1}, 1\right)-\mu_{W}\left(1-z_{1}\right) F_{0}^{W}(1)
$$

Define $\alpha\left(z_{1}\right)=\lambda_{1}\left(1-z_{1}\right)+\gamma+\mu_{P}\left(1-\frac{1}{z_{1}}\right)$ and $\beta\left(z_{1}\right)=\left(\lambda_{1} z_{1}-\mu_{W}\right)\left(1-z_{1}\right)+\eta z_{1}$. Then, (16) and (17) can be rewritten as

$$
\xi\left(1-z_{1}\right) \frac{\partial F_{P}\left(z_{1}, 1\right)}{\partial z_{1}}=\alpha\left(z_{1}\right) F_{P}\left(z_{1}, 1\right)-\mu_{P}\left(1-\frac{1}{z_{1}}\right) F_{0}^{P}(1)-\eta F_{W}\left(z_{1}, 1\right)
$$

and

$$
\beta\left(z_{1}\right) F_{W}\left(z_{1}, 1\right)=\gamma z_{1} F_{P}\left(z_{1}, 1\right)-\mu_{W}\left(1-z_{1}\right) F_{0}^{W}(1)
$$

Also, $z_{1,1}$ and $z_{1,2}$ are the roots of $\beta\left(z_{1}\right)$, which are

$$
z_{1,1}=\frac{\lambda_{1}+\mu_{W}+\eta-\sqrt{\left(\lambda_{1}+\mu_{W}+\eta\right)^{2}-4 \lambda_{1} \mu_{W}}}{2 \lambda_{1}}
$$

and

$$
z_{1,2}=\frac{\lambda_{1}+\mu_{W}+\eta+\sqrt{\left(\lambda_{1}+\mu_{W}+\eta\right)^{2}-4 \lambda_{1} \mu_{W}}}{2 \lambda_{1}}
$$

Finally, substituting (19) in (18) leads to the following differential equation,

$$
\frac{\partial F_{P}\left(z_{1}, 1\right)}{\partial z_{1}}-\frac{\alpha\left(z_{1}\right) \beta\left(z_{1}\right)-\eta \gamma z_{1}}{\xi\left(1-z_{1}\right) \beta\left(z_{1}\right)} F_{P}\left(z_{1}, 1\right)=\frac{\eta \mu_{W} F_{0}^{W}(1)}{\xi \beta\left(z_{1}\right)}-\frac{\mu_{P} F_{0}^{P}(1)}{\xi z_{1}}
$$

We introduce the function $f\left(z_{1}\right)=-\frac{\alpha\left(z_{1}\right) \beta\left(z_{1}\right)-\eta \gamma z_{1}}{\xi\left(1-z_{1}\right) \beta\left(z_{1}\right)}$. Performing some simple calculused operations, $f\left(z_{1}\right)$ transforms into

$$
f\left(z_{1}\right)=-\frac{\lambda_{1}}{\xi}+\frac{\mu_{P}}{\xi z_{1}}+\frac{\gamma}{\xi\left(1-z_{1}\right)}\left(\frac{\eta z_{1}}{\beta\left(z_{1}\right)}-1\right)
$$

After some algebra and applying the partial fraction, expansion the function $f\left(z_{1}\right)$ becomes

$$
f\left(z_{1}\right)=-\frac{\lambda_{1}}{\xi}+\frac{\mu_{P}}{\xi z_{1}}+\frac{\gamma}{\xi}\left(\frac{M}{z_{1}-z_{1,1}}+\frac{N}{z_{1,2}-z_{1}}\right)
$$


where $M=\left.\frac{\frac{\mu_{W}}{\lambda_{1}}-z_{1}}{z_{1,2}-z_{1}}\right|_{z_{1}=z_{1,1}}=\frac{\frac{\mu_{W}}{\lambda_{1}}-z_{1,1}}{z_{1,2}-z_{1,1}}=\frac{z_{1,1} z_{1,2}-z_{1,1}}{z_{1,2}-z_{1,1}}>0$ and $\quad N=\left.\frac{\frac{\mu_{W}}{\lambda_{1}}-z_{1}}{z_{1}-z_{1,1}}\right|_{z_{1}=z_{1,2}}=\frac{z_{1,1} z_{1,2}-z_{1,2}}{z_{1,2}-z_{1,1}}$ $<0$. In order to solve the differential equation (20), we can multiply it by $e^{\int f\left(z_{1}\right) d z_{1}}$. Hence, we get

$$
\frac{\partial F_{P}\left(z_{1}, 1\right)}{\partial z_{1}} e^{\int f\left(z_{1}\right) d z_{1}}+f\left(z_{1}\right) e^{\int f\left(z_{1}\right) d z_{1}} F_{P}\left(z_{1}, 1\right)=\left[\frac{\eta \mu_{W} F_{0}^{W}(1)}{\xi \beta\left(z_{1}\right)}-\frac{\mu_{P} F_{0}^{P}(1)}{\xi z_{1}}\right] e^{\int f\left(z_{1}\right) d z_{1}}
$$

The function in (22) is integrated as

$$
\int f\left(z_{1}\right) d z_{1}=-\frac{\lambda_{1}}{\xi} z_{1}+\frac{\mu_{P}}{\xi} \ln \left|z_{1}\right|+\frac{\gamma M}{\xi} \ln \left|z_{1}-z_{1,1}\right|-\frac{\gamma N}{\xi} \ln \left(z_{1,2}-z_{1}\right)
$$

Therefore, we have

$$
e^{\int f\left(z_{1}\right) d z_{1}}=e^{-\frac{\lambda_{1} z_{1}}{\xi}} z_{1}^{\frac{\mu_{P}}{\xi}}\left|z_{1}-z_{1,1}\right|^{\frac{\gamma M}{\xi}}\left(z_{1,2}-z_{1}\right)^{-\frac{\gamma N}{\xi}}
$$

Then, (23) is equal to

$$
\frac{\partial}{\partial z_{1}}\left[e^{-\frac{\lambda_{1}}{\xi}}\left|z_{1}-z_{1,1}\right|^{\frac{\gamma M}{\xi}} z_{1}^{\frac{\mu_{P}}{\xi}}\left(z_{1,2}-z_{1}\right)^{-\frac{\gamma N}{\xi}} F_{P}\left(z_{1}, 1\right)\right]=\left[\frac{\eta \mu_{W} F_{0}^{W}(1)}{\xi \beta\left(z_{1}\right)}-\frac{\mu_{P} F_{0}^{P}(1)}{\xi z_{1}}\right] e^{\int f\left(z_{1}\right) d z_{1}}
$$

where $k_{1}\left(z_{1}\right)$ and $k_{2}\left(z_{1}\right)$ are defined as

and

$$
k_{1}\left(z_{1}\right)=e^{-\frac{\lambda_{1}}{\xi} z_{1}}\left(z_{1,1}-z_{1}\right)^{\frac{\gamma M}{\xi}} z_{1}^{\frac{\mu_{p}}{\xi}}\left(z_{1,2}-z_{1}\right)^{-\frac{\gamma N}{\xi}}, z_{1} \leq z_{1,1}
$$

$$
k_{2}\left(z_{1}\right)=e^{-\frac{\lambda_{1}}{\xi} z_{1}}\left(z_{1}-z_{1,1}\right)^{\frac{\gamma M}{\xi}} z_{1}^{\frac{\mu_{p}}{\xi}}\left(z_{1,2}-z_{1}\right)^{-\frac{\gamma N}{\xi}}, z_{1} \geq z_{1,1}
$$

Then, (26) becomes

And after integrating, we obtain

$$
\begin{aligned}
& \frac{\partial}{\partial z_{1}}\left[k_{1}\left(z_{1}\right) F_{P}\left(z_{1}, 1\right)\right]=\left[\frac{\eta \mu_{W} F_{0}^{W}(1)}{\xi \beta\left(z_{1}\right)}-\frac{\mu_{P} F_{0}^{P}(1)}{\xi z_{1}}\right] k_{1}\left(z_{1}\right), z_{1} \leq z_{1,1} \\
& \frac{\partial}{\partial z_{1}}\left[k_{2}\left(z_{1}\right) F_{P}\left(z_{1}, 1\right)\right]=\left[\frac{\eta \mu_{W} F_{0}^{W}(1)}{\xi \beta\left(z_{1}\right)}-\frac{\mu_{P} F_{0}^{P}(1)}{\xi z_{1}}\right] k_{2}\left(z_{1}\right), z_{1} \geq z_{1,1}
\end{aligned}
$$

$$
\begin{aligned}
& k_{1}\left(z_{1}\right) F_{P}\left(z_{1}, 1\right)=\frac{\eta \mu_{W} F_{0}^{W}(1)}{\xi} \int_{0}^{z_{1}} \frac{k_{1}(x)}{\beta(x)} d x-\frac{\mu_{P} F_{0}^{P}(1)}{\xi} \int_{0}^{z_{1}} \frac{k_{1}(x)}{x} d x+C_{1}, z_{1} \leq z_{1,1} \\
& k_{2}\left(z_{1}\right) F_{P}\left(z_{1}, 1\right)=\frac{\eta \mu_{W} F_{0}^{W}(1)}{\xi} \int_{z_{1,1}}^{z_{1}} \frac{k_{2}(x)}{\beta(x)} d x-\frac{\mu_{P} F_{0}^{P}(1)}{\xi} \int_{z_{1,1}}^{z_{1}} \frac{k_{2}(x)}{x} d x+C_{2}, z_{1} \geq z_{1,1}
\end{aligned}
$$

We need to determine the coefficients $C_{1}$ and $C_{2}$. Let's $z_{1}=0$ in (31), we get $k_{1}(0)=$ $z_{1,1}^{\frac{\gamma M}{\xi}} z_{1,2}^{-\frac{\gamma N}{\xi}}$. And knowing $F_{P}(0,1)=F_{0}^{P}(1)$, we get $C_{1}=z_{1,1}^{\frac{\gamma M}{\xi}} z_{1,2}^{-\frac{\gamma N}{\xi}} F_{0}^{P}(1)$. In a similar fashion we get $C_{2}=0$. Finally, while the PGF in the PLC state, we have 


$$
\begin{gathered}
F_{P}\left(z_{1}, 1\right)=\frac{\frac{\eta \mu_{W} F_{0}^{W}(1)}{\xi} \int_{0}^{z_{1}} \frac{k_{1}(x)}{\beta(x)} d x-\frac{\mu_{P} F_{0}^{P}(1)}{\xi} \int_{0}^{z_{1}} \frac{k_{1}(x)}{x} d x+z_{1,1}^{\frac{\gamma M}{\xi}} z_{1,2}^{-\frac{\gamma N}{\xi}} F_{0}^{P}(1)}{k_{1}\left(z_{1}\right)}, z_{1} \leq z_{1,1} \\
F_{P}\left(z_{1}, 1\right)=\frac{\frac{\eta \mu_{W} F_{0}^{W}(1)}{\xi} \int_{z_{1,1}}^{z_{1}} \frac{k_{2}(x)}{\beta(x)} d x-\frac{\mu_{P} F_{0}^{P}(1)}{\xi} \int_{z_{1,1}}^{z_{1}} \frac{k_{2}(x)}{x} d x}{k_{2}\left(z_{1}\right)}, z_{1} \geq z_{1,1}
\end{gathered}
$$

In the last equation, the "zero probabilities" we can find them in the following way, we know that

$$
\pi_{P}=\frac{\eta}{\eta+\gamma}=F_{P}(1,1)=\frac{\frac{\eta \mu_{W} F_{0}^{W}(1)}{\xi} \int_{z_{1,1}}^{1} \frac{k_{2}(x)}{\beta(x)} d x-\frac{\mu_{P} F_{0}^{P}(1)}{\xi} \int_{z_{1,1}}^{1} \frac{k_{2}(x)}{x} d x}{k_{2}(1)}
$$

Similarly, from the boundary conditions in (33) for $z_{1}=z_{1,1}$, we get

$$
\frac{\eta \mu_{W} F_{0}^{W}(1)}{\xi} \int_{0}^{z_{1,1}} \frac{k_{1}(x)}{\beta(x)} d x-\frac{\mu_{P} F_{0}^{P}(1)}{\xi} \int_{0}^{z_{1,1}} \frac{k_{1}(x)}{x} d x+z_{1,1}^{\frac{\gamma M}{\xi}} z_{1,2}^{-\frac{\gamma N}{\xi}} F_{0}^{P}(1)=0
$$

Define $S=\int_{0}^{z_{1,1}} \frac{k_{1}(x)}{\beta(x)} d x, T=\int_{0}^{z_{1,1}} \frac{k_{1}(x)}{x} d x, U=\int_{z_{1,1}}^{1} \frac{k_{2}(x)}{\beta(x)} d x, V=\int_{z_{1,1}}^{1} \frac{k_{2}(x)}{x} d x$ and $R=$ $z_{1,1}^{\frac{\gamma M}{\xi}} z_{1,2}^{-\frac{\gamma N}{\xi}}$. Then, (35) and (36) can be rewritten as

Then, we get

$$
\begin{gathered}
\frac{\eta}{\eta+\gamma}=\frac{\frac{\eta \mu_{W} F_{0}^{W}(1)}{\xi} U-\frac{\mu_{P} F_{0}^{P}(1)}{\xi} V}{k_{2}(1)} \\
\frac{\eta \mu_{W} F_{0}^{W}(1)}{\xi} S-\frac{\mu_{P} F_{0}^{P}(1)}{\xi} T+R F_{0}^{P}(1)=0
\end{gathered}
$$

$$
\begin{gathered}
F_{0}^{P}(1)=\frac{S \eta \xi k_{2}(1)}{(\eta+\gamma)\left[\left(\mu_{P} T-R S\right) U-\mu_{P} V S\right]} \\
F_{0}^{W}(1)=\frac{\left(\mu_{P} T-R S\right) \xi k_{2}(1)}{\mu_{W}(\eta+\gamma)\left[\left(\mu_{P} T-R S\right) U-\mu_{P} V S\right]}
\end{gathered}
$$

Differentiating (14) and (15) with respecting to $z_{1}$, we get

$$
\begin{aligned}
& \lambda_{1}\left(1-z_{1}\right) \frac{\partial F_{P}\left(z_{1}, 1\right)}{\partial z_{1}}-\lambda_{1} F_{P}\left(z_{1}, 1\right)+\gamma \frac{\partial F_{P}\left(z_{1}, 1\right)}{\partial z_{1}}+\mu_{P}\left(1-\frac{1}{z_{1}}\right) \frac{\partial F_{P}\left(z_{1}, 1\right)}{\partial z_{1}}+\frac{\mu_{P}}{z_{1}^{2}} F_{P}\left(z_{1}, 1\right) \\
& +\xi\left(z_{1}-1\right) \frac{\partial^{2} F_{P}\left(z_{1}, 1\right)}{\partial z_{1}^{2}}+\xi \frac{\partial F_{P}\left(z_{1}, 1\right)}{\partial z_{1}}=\eta \frac{\partial F_{W}\left(z_{1}, 1\right)}{\partial z_{1}}+\frac{\mu_{P}}{z_{1}^{2}} F_{0}^{P}(1) \\
& {\left[\lambda_{1}\left(1-z_{1}\right)+\eta\right] \frac{\partial F_{W}\left(z_{1}, 1\right)}{\partial z_{1}}+\mu_{W} \frac{1}{z_{1}^{2}}\left[F_{W}\left(z_{1}, 1\right)-F_{0}^{W}(1)\right]+\mu_{W}\left(1-\frac{1}{z_{1}}\right) \frac{\partial F_{W}\left(z_{1}, 1\right)}{\partial z_{1}}} \\
& =\lambda_{1} F_{W}\left(z_{1}, 1\right)+\gamma \frac{\partial F_{P}\left(z_{1}, 1\right)}{\partial z_{1}}
\end{aligned}
$$


Taking $z_{1}=1$ into (41) and (42), we get

$$
\begin{gathered}
(\xi+\gamma) L_{1 P}+\left(\mu_{P}-\lambda_{1}\right) \pi_{P}=\eta L_{1 W}+\mu_{P} F_{0}^{P}(1) \\
\eta L_{1 W}+\mu_{W}\left[\pi_{W}-F_{0}^{W}(1)\right]=\lambda_{1} \pi_{W}+\gamma L_{1 P}
\end{gathered}
$$

where $L_{1 P}=\left.\frac{\partial F_{P}\left(z_{1}, 1\right)}{\partial z_{1}}\right|_{z_{1}=1}, L_{1 W}=\left.\frac{\partial F_{W}\left(z_{1}, 1\right)}{\partial z_{1}}\right|_{z_{1}=1}, F_{P}(1,1)=\pi_{P}, F_{W}(1,1)=\pi_{W}$. Then, we get

$$
\begin{gathered}
L_{1 P}=\frac{\lambda_{1}-\mu_{W}\left[\pi_{W}-F_{0}^{W}(1)\right]-\mu_{P}\left[\pi_{P}-F_{0}^{P}(1)\right]}{\eta} \\
L_{1 W}=\frac{\lambda_{1}\left(\eta \pi_{W}+\gamma\right)-(\gamma+\eta) \mu_{W}\left[\pi_{W}-F_{0}^{W}(1)\right]-\gamma \mu_{P}\left(\pi_{P}-F_{0}^{p}(1)\right)}{\eta^{2}}
\end{gathered}
$$

So far, we can derive the average number of the low priority data in the queuing model $L_{1}=L_{1 P}+L_{1 W}$. Finally, using the Little's Law $L_{1}=\lambda_{1} E\left(T_{1}\right)$, Theorem 1 is proved.

Theorem 2 . The mean transmission delay of the high priority data in the queuing model is

$$
E\left(T_{2}\right)=\frac{L_{2 P}+L_{2 W}}{\lambda_{2}}=\frac{1}{\lambda_{2}}\left[\frac{\lambda_{2}-\mu_{W}\left[F_{0}^{W}(1)-\pi_{0,0, W}\right]}{\xi}\right]+\frac{1}{\lambda_{2}}\left[\frac{(\xi+\gamma) L_{2 P}-\lambda_{2} P_{P}}{\eta}\right]
$$

Proof: we take $z_{1}=1$ into the (12) and (13), and then we have

$$
\begin{gathered}
\lambda_{2}\left(1-z_{2}\right) F_{P}\left(1, z_{2}\right)+\gamma F_{P}\left(1, z_{2}\right)+\xi\left(z_{2}-1\right) \frac{\partial F_{P}\left(1, z_{2}\right)}{\partial z_{2}}=\eta F_{W}\left(1, z_{2}\right) \\
\lambda_{2} z_{2} F_{W}\left(1, z_{2}\right)+\gamma F_{P}\left(1, z_{2}\right)=\left(\lambda_{2}+\eta\right) F_{W}\left(1, z_{2}\right)+\mu_{W}\left(1-\frac{1}{z_{2}}\right)\left[F_{0}^{W}\left(z_{2}\right)-\pi_{0,0, W}\right]
\end{gathered}
$$

Differentiating (48) and (49) with respecting to $z_{2}$ and taking $z_{2}=1$, we get

$$
\begin{gathered}
(\gamma+\xi) L_{2 p}=\eta L_{2 W}+\lambda_{2} P_{P} \\
\eta L_{2 W}+\mu_{W}\left[F_{0}^{W}(1)-\pi_{0,0, W}\right]=\lambda_{2} P_{W}+\gamma L_{2 P}
\end{gathered}
$$

where $L_{2 P}=\frac{\lambda_{2}-\mu_{W}\left[F_{0}^{W}(1)-\pi_{0,0, W}\right]}{\xi}, L_{2 W}=\frac{(\xi+\gamma) L_{2 P}-\lambda_{2} P_{P}}{\eta}, \pi_{0,0, W}$ means the initial probability of finding no data in the WiFi state. Thus, the law of the total probability can be written as $\pi_{0,0, W}=\pi_{W}(i=0, j=0)=\pi_{W}(j=0 / i=0) \pi_{W}(i=0)$. Here, $\pi_{W}(i=0)=F_{0}^{W}(1)$. $\pi_{W}(j=0 / i=0)$ means no the low priority data in the system. Thus, the high priority data is similar to the low priority and the service process which can be described as the 2D Markov chain with FCFS depicted as shown in [30]. Thus, $\pi_{W}(j=0 / i=0)=\pi_{2 W}(j=0)$, while $i=0$. According to the experimental in [30], $\pi_{2 W}(j=0)$ is obtained

$$
\begin{gathered}
\pi_{2 W}(j=0)=\frac{\xi m_{2}(1)}{(\eta+\gamma) \mu_{W}} \frac{1}{\int_{y_{1}}^{1} \frac{m_{2}(z)}{\beta(z)} d z} \\
\pi_{2 P}(j=0)=\frac{\xi m_{2}(1) \int_{0}^{y_{1}} \frac{m_{1}(z)}{\beta(z)} d z}{(\eta+\gamma) y_{1}^{\frac{\gamma K}{\xi}} y_{2}^{\frac{\gamma J}{\xi}} \int_{y_{1}}^{1} \frac{m_{2}(z)}{\beta(z)} d z}
\end{gathered}
$$


where

$$
\begin{aligned}
& m_{1}\left(z_{1}\right)=e^{-\frac{\lambda_{2}}{\xi} z_{1}}\left(y_{1}-z\right)^{\frac{\gamma K}{\xi}}+\left(y_{2}-z_{1}\right)^{-\frac{\gamma J}{\xi}}, z \leq y_{1} \\
& m_{2}\left(z_{1}\right)=e^{-\frac{\lambda_{2}}{\xi} z_{1}}\left(z-y_{1}\right)^{\frac{\gamma K}{\xi}}+\left(y_{2}-z_{1}\right)^{-\frac{\gamma J}{\xi}}, z \geq y_{1}
\end{aligned}
$$

where $y_{1}$ and $y_{2}\left(0<y_{1}<1<y_{2}\right)$ are the roots of $g(z)=-\frac{\left[\lambda_{2}(1-z)+\gamma\right] \alpha(z)-\eta \gamma z}{\xi(1-z) \alpha(z)}$. Here, $\alpha(z)=\left(\lambda_{2} z-\mu_{w}\right)(1-z)+\eta z$. The coefficient $K=\frac{y_{1} y_{2}-y_{1}}{y_{2}-y_{1}}>0$ and $J=\frac{y_{1} y_{2}-y_{2}}{y_{2}-y_{1}}<0$.

Then, we get

$$
\pi_{0,0, W}=\frac{\xi m_{2}(1)}{(\eta+\gamma) \mu_{W}} \frac{1}{\int_{y_{1}}^{1} \frac{m_{2}(z)}{\beta(z)} d z} F_{0}^{W}(1)
$$

Now, the mean transmission delay of the high priority data in the queuing model is $L_{2}=L_{2 P}+L_{2 W}$. Finally, we obtain the mean transmission delay of the high priority as in (25) by using the Little's Law $L_{2}=\lambda_{2} E\left(T_{2}\right)$, then we get (47).

Corollary 1. The mean transmission delay of the low priority in the PLC state and WiFi state are

$$
\begin{gathered}
E\left(T_{1 P}\right)=\frac{L_{1 P}}{\lambda_{1}}=\frac{\lambda_{1}-\mu_{W}\left[\pi_{W}-F_{0}^{W}(1)\right]-\mu_{P}\left[\pi_{P}-F_{0}^{P}(1)\right]}{\lambda_{1} \eta} \\
E\left(T_{1 W}\right)=\frac{L_{1 W}}{\lambda_{1}}=\frac{\lambda_{1}\left(\eta \pi_{W}+\gamma\right)-(\gamma+\eta) \mu_{W}\left[\pi_{W}-F_{0}^{W}(1)\right]-\gamma \mu_{P}\left[\pi_{P}-F_{0}^{P}(1)\right]}{\lambda_{1} \eta^{2}}
\end{gathered}
$$

Similarly, the mean transmission delays of the high priority in the PLC state and the WiFi state are

$$
\begin{gathered}
E\left(T_{2 P}\right)=\frac{L_{2 P}}{\lambda_{2}}=\frac{\lambda_{2}-\mu_{W}\left[F_{0}^{W}(1)-\pi_{0,0, W}\right]}{\lambda_{2} \xi} \\
E\left(T_{2 W}\right)=\frac{L_{2 W}}{\lambda_{2}}=\frac{(\xi+\gamma) L_{2 P}-\lambda_{2} P_{P}}{\lambda_{2} \eta}
\end{gathered}
$$

\subsection{Attractor Selection Model}

The original attractor selection model (ASM), which has two attractors corresponding to two proteins, can adaptively change the external form of the cell according to the external environment. The ASM was applied in network research in [31]-[33], because of the similarity between the network selection adapted to the network environment and the cells adapted to the external environment. Therefore, the network configuration, which represents the operator in the original ASM, achieves the purpose of selecting the nearest network path by controlling of the operator selection. We map the variables of the network, which are network performance parameters including the network time delay, throughput, packet loss rate etc., to the attractors. And then the network can adjust the allocation of the resources according to the environment.

According to [33], the ASM is expressed in mathematical formulas which can be written as 


$$
\left\{\begin{array}{c}
\frac{d m_{1}}{d t}=\frac{s(A)}{1+m_{*}^{2}-m_{1}^{2}}-d(A) * m_{1}+\varepsilon_{1} \\
\frac{d m_{2}}{d t}=\frac{s(A)}{1+m_{*}^{2}-m_{2}^{2}}-d(A) * m_{2}+\varepsilon_{2} \\
m_{*}=\max \left\{m_{1}, m_{2}\right\}
\end{array}\right.
$$

where $m_{1}$ and $m_{2}$, transcribed from Operon 1 and Operon 2, are the concentrations of the two mRNAs, respectively. $A$ is the cell activity, which is used to quantify cell growth and to capture the phenotypic consequence. That is to say, the value of the cell activity is a measure of the adaptation of the cells to the environment. Thus, the higher cell activity, the more adapted the cell is to the environment and the more stable the cell state is. In the network path selection researches, we can map the network environment to $A$. Moreover, $s(A)$ and $d(A)$ are the rate coefficients of synthesis and degradation (dilution), respectively. (61) means that the value of $s(A)$ is high, while the system state is suitable for the environment well and vice versa. $s(A)$ and $d(A)$ are monotonously increased nonnegative function of $A . \varepsilon_{1}$ and $\varepsilon_{2}$ denote the internal noises in the gene expression, which are independent of each other. The variation of the selection probabilities over the parameter $A$ is shown in Fig. 6.

\subsection{Extended Attractor Selection Model}

According to the original ASM, the extended ASM is established to use in the network environment. Thus, we use the following extended ASM to select the optimal path (PLC or $\mathrm{WiFi}$ ), to maximize the value of activity defined by the queue length of the target objects. According to (61), we can get the fixed-equilibrium solution of the system as follows:

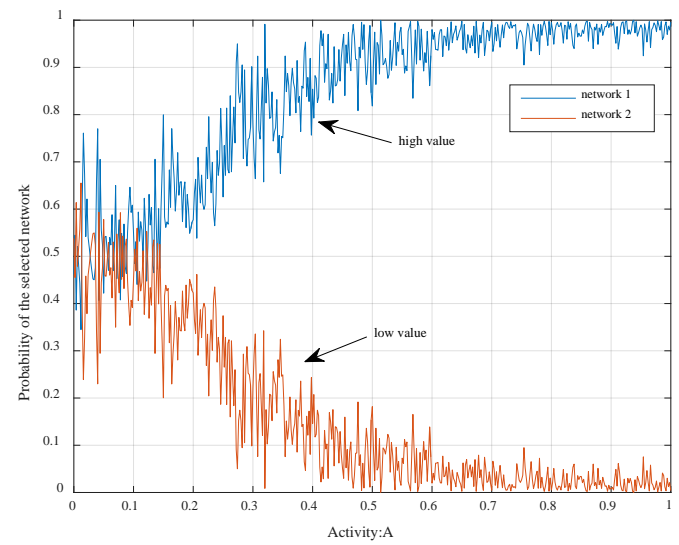

Fig. 6. The variation of the selection probabilities over the parameter $A$

$$
\left\{\begin{array}{c}
\frac{s(A)}{1+m_{*}^{2}-m_{l}^{2}}-d(A) \times m_{l}=0 \\
s(A)-d(A) \times m_{l}=0 \\
m_{*}=\max \left\{m_{l}\right\} \\
l=1,2
\end{array}\right.
$$


We define $s(A)=A\left[a A^{b}+\frac{1}{\sqrt{2}}\right]$, and $d(A)=A$, where parameters $a$ and $b$ are the factors which can be set as $a=5, b=2$. For the sake of simplicity, we denote $\Phi(A)=\frac{s(A)}{d(A)}$, then (62) can be rewritten as

$$
m_{l}=\left\{\begin{array}{cc}
\Phi(A) & \text { high-value } \\
\frac{1}{2}\left[\sqrt{4+\Phi(A)^{2}}-\Phi(A)\right] & \text { low-value }
\end{array}\right.
$$

From (63), the selection probability converges into two states, one is high-value and the other is low-value. In this paper, we assume the set of selection including the PLC or WiFi. That is to say, a node in the heterogeneous IoT chooses one of the two paths, i.e. the PLC or $\mathrm{WiFi}$, to maximize the activity defined by the mean transmission delay of the target network. Then, the multipath selection process is shown in Fig. 7. In Fig. 7, there are two paths between the every two nodes. If a path or node is not suitable for transmitting data, the optimal path is switched to the best secondary path automatically.

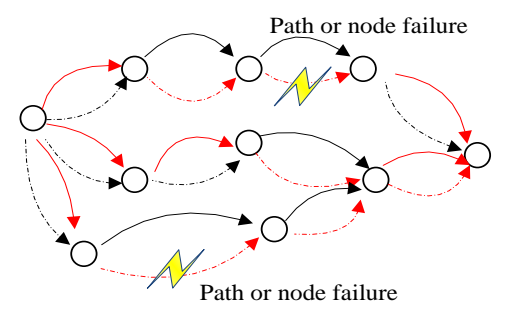

(a) Failure of primary path

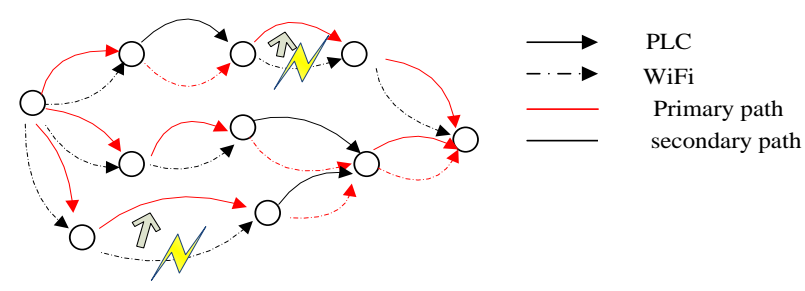

(b) Switching to secondary path

Fig. 7. The multipath selection process

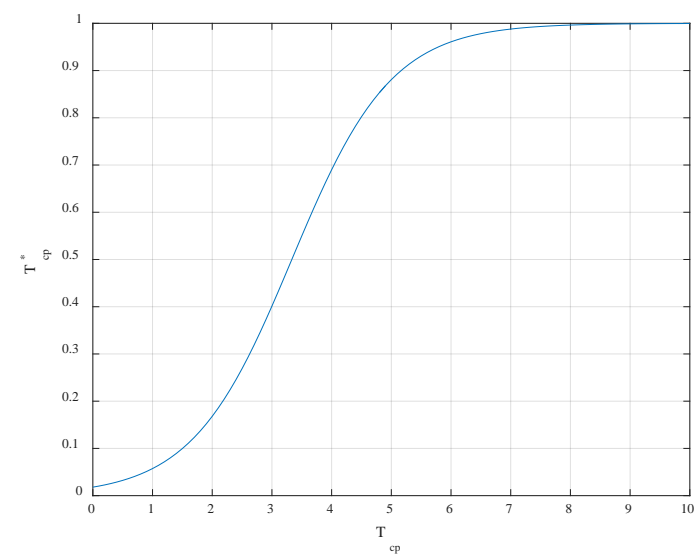

Fig. 8. The relationship between $T_{C P}$ and $T_{C P}^{*}$

\section{Mapping A to the Activity}

In this section, we provide the activity expression of the heterogeneous IoT network. Our goal is to select an optimal path according to the mean transmission delay of the different priorities. That is to say, the heterogeneous IoT network applies the attractor selection algorithm to select the PLC or WiFi to transmit data, which has the shorter mean transmission delay. The proposed algorithm combing the 3D Markov chain and the ASM is named MASM. 
Based on the above analysis, the heterogeneous IoT environment is related to the mean transmission delay in the PLC or WiFi network. We assume $T_{C P} \in T=\left\{E\left(T_{1 P}\right), E\left(T_{1 W}\right), E\left(T_{2 P}\right)\right.$ $\left.E\left(T_{2 W}\right)\right\}$. Here, $T_{C P}$ is the transmission delay of the current path (CP). Thus, we map the mean transmission delay into $A$ which can represent the network environment (queue length or delay) as

$$
A=1-\frac{1}{1+e^{-\kappa\left[T_{C P}^{*}-\Delta\right]}}
$$

where $T_{C P}^{*}$ is the normalized result of $T_{C P}$. The relationship between $T_{C P}^{*}$ and $T_{C P}$ is shown in Fig. 8. $\kappa$ is the constant which influences the mapping of the activity. Fig. 9 shows the relationship between $A$ and $\kappa$, which can be found that $\kappa$ can be used to control the steepness of the shape of $A$. The larger parameters $\kappa$ are, the steeper slope of $A$ becomes. Hence, $\kappa$ is allowed to tune and control the sensitivity of $A$, while $\kappa$ approaches to 1 or decreases to 0 . The relationship between $A$ and $\Delta$ which is a threshold value is shown in Fig. 10. When $\Delta$ is too large, $A$ becomes slow in response to the metric modification and the performance degradation. Hence, exploiting the sensitivity of $A$ to control the parameter $\kappa$ and $\Delta$, we simply adopt the specific setting $\{\Delta=0.4, \kappa=10\}$ for the MASM.

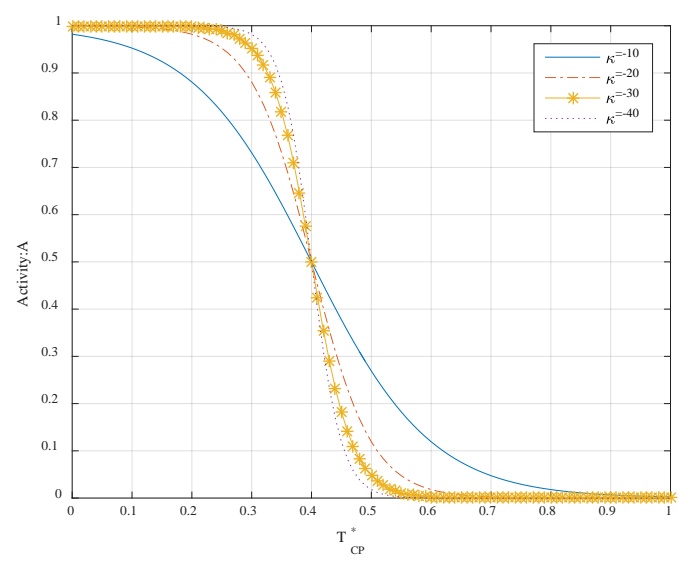

Fig. 9. Different shapes of $A$ with different settings on $\kappa$

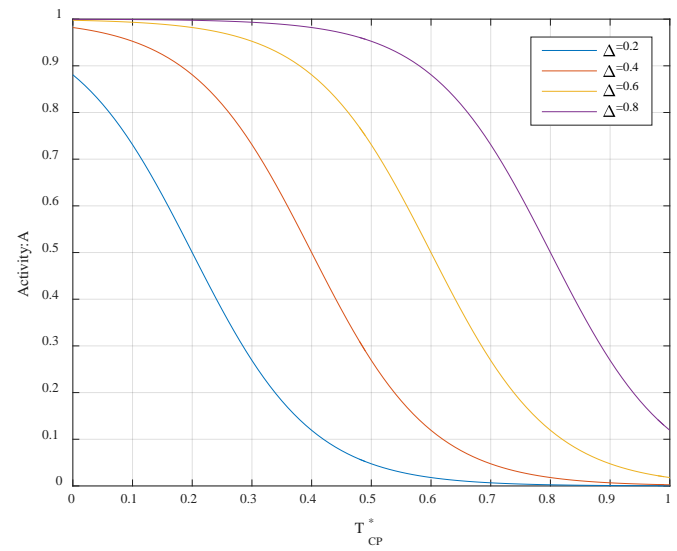

Fig. 10. Different shapes of $A$ with different settings on $\Delta$ 


\section{Simulation and Numerical Analysis}

In this section, the performances comparison (including time delay, delivery ratio,etc.) of the MASM, AODV, GA, SAW and ISRO_PG are proposed. Firstly, the parameters are initialized. Then, the simulation results confirme that the MASM is superior to others by compared the experiments.

\subsection{Validation of Queuing Model}

In this subsection, we evaluate the multi-priority queuing model and the theory results by simulation. According to [33], we assume data rate for PLC and WiFi is $20 \mathrm{Mb} / \mathrm{s}$. Unless otherwise stated, the deadlines of the different priorities are limited independent exponentially distributed. Moreover, the durations of the PLC and WiFi availability and unavailability periods are independent exponential distributions with rate $\eta$ and $\gamma$, respectively. At the same time, data sizes of the different priorities are independent exponentially distributed, and data arrival of the different priorities are Poisson process with rate $\lambda_{1}$ and $\lambda_{2}$, respectively. The mean data size for the each classes is assumed to be 2MB [33]. For simplicity, the performances of our simulations are tested for both mobile and fixed scenarios, and some useful notations are shown in Table 2.

Table 2. Variable and the value

\begin{tabular}{|c|c|c|}
\hline Variable & Value in the fixed scenario & Value in the mobile scenario \\
\hline$\mu_{P}$ & $1 / 150 \mathrm{~s}^{-1}$ & $1 / 30 \mathrm{~s}^{-1}$ \\
\hline$\mu_{W}$ & $1 / 600 \mathrm{~s}^{-1}$ & $1 / 300 \mathrm{~s}^{-1}$ \\
\hline$\eta$ & 0.5 & 0.5 \\
\hline$\gamma$ & 0.5 & 0.5 \\
\hline
\end{tabular}

The queuing model is consist of the fixed scenario and the mobile scenario. Fig. 11 shows the mean transmission delay in the fixed scenario, and Fig. 12 shows the mean transmission delay in the mobile scenario. As shown in these two figures, there are a good match between the theory and the simulation results. And the value of the mean transmission delay increases larger, if data arrival rate becomes larger. That is, the mean transmission delay as expected is increased by increasing the arrival rate owning to the slow service.
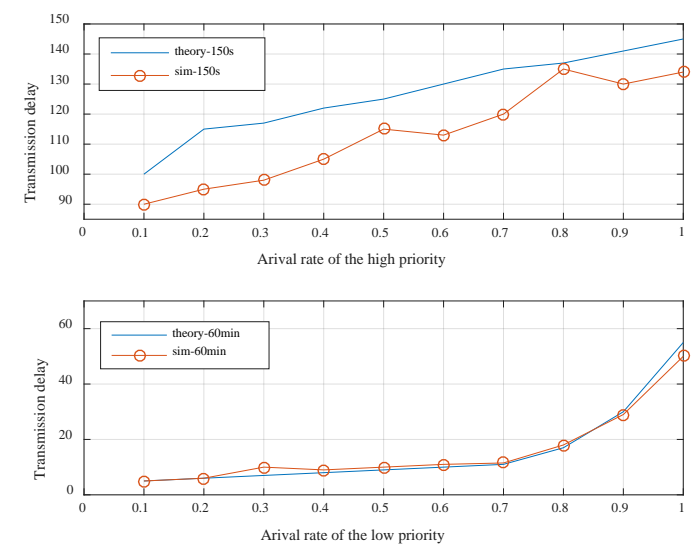

Fig. 11. The mean transmission delay for the fixed scenario 

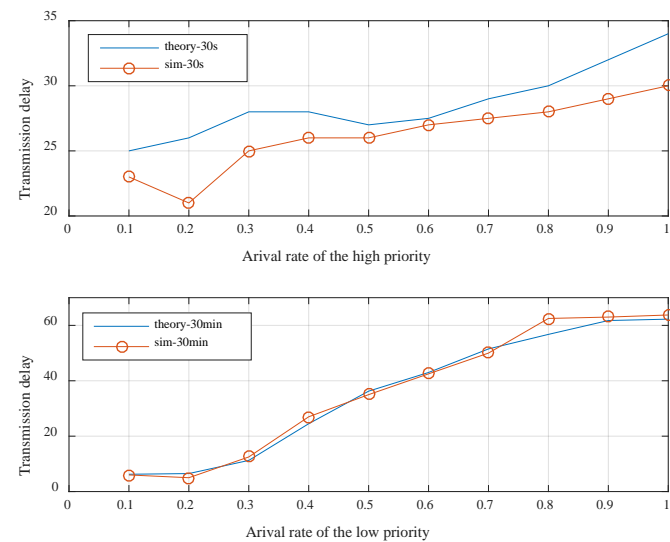

Fig. 12. The mean transmission delay for the mobile scenario

\subsection{Implementation of the Adaptive Network Selection based on the Attractor Selection}

In this subsection, the MASM is compared with the AODV, GA, SAW and ISRO_PG. The AODV is a well-known reactive routing protocol which determines unicast routes to destinations with the advantaged of the dynamic link conditions, low processing and overhead. The GA can select the best choice available at every step without regard for the possible future consequences. The SAW, which is widely used in the recent researches [33], [34], is one of methods of MADM. And the ISRO_PG based on the Game Theory which is also widely used in the recent researches for the network selection problems [35], [36]. The QoS requirements of the applications assumed in the SAW and ISRO_PG are references to [33], [35], respectively.

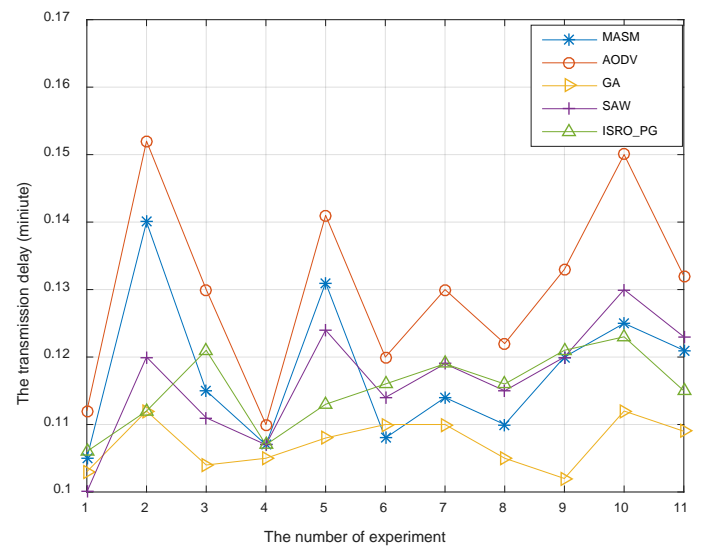

Fig. 13. The transmission delay 


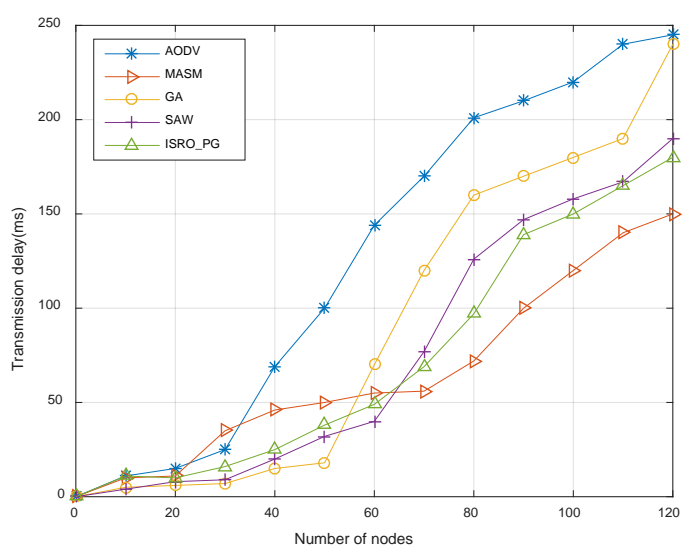

Fig. 14. The transmission delay against the number of nodes

Firstly, 20 nodes are distributed in the heterogeneous IoT. Each node can communicate with the node within the distance at data rate of 2Mbps by using IEEE 802.11b. Fig. 13 shows the simulation results. The $\mathrm{x}$-axis is the number of the experiments. The mean delay values of these five networks differ very little. Then the nodes are increased from 10 to 120 for the real heterogeneous IoT environment. Fig. 14 shows the mean transmission delay comparison between the MASM, AODV, GA, SAW and ISRO_PG with the different number of nodes. With the number of nodes increasing, the mean transmission delay of these five algorithms increase accordingly. Specifically, while the number of nodes increases, the mean transmission delay of the MASM increases relatively little. It is because that the MASM can choose the optimal path to reduce the mean transmission delay dynamically. The ISRO_PG also allocates the network resources according to the environment. However, the computation times of the ISRO_PG increase with the number of nodes increasing. Thus, the mean transmission delay increases in the ISRO_PG scenario.

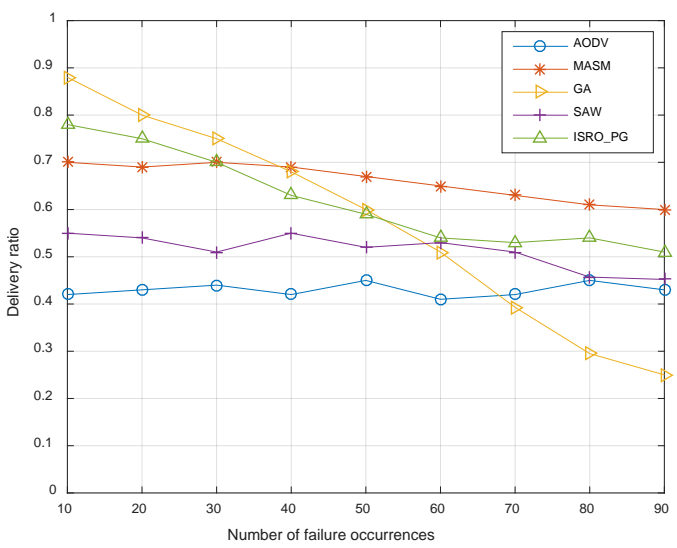

Fig. 15. The delivery ratio against failures 


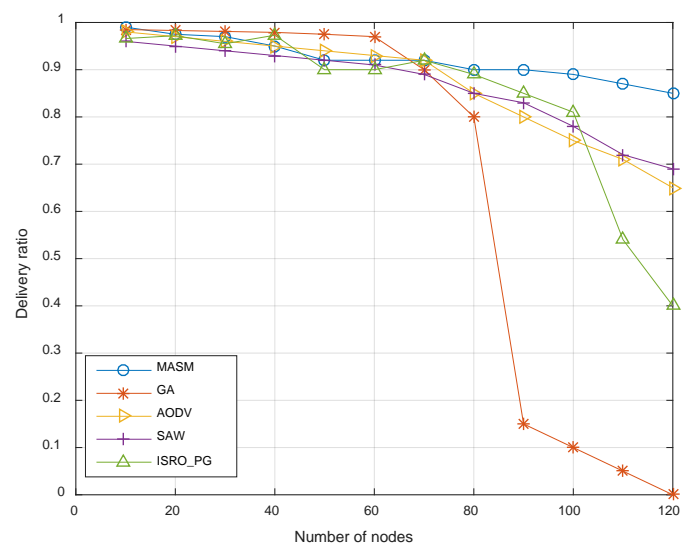

Fig. 16. The delivery ratio against the number of nodes

The delivery ratio against failures is illustrated in Fig. 15. As shown in the figure, the MASM achieves higher delivery ratio than that of AODV and SAW for all the scenarios. The GA provides the higher delivery ratio under the less dynamic conditions, but the performance gradual decreases with the number of the failure occurrence increasing. While the number of occurrence exceeds 70 , the performance of the GA decreases to the lowest in these five algorithms. It is because that GA introduces excessive overhead in the path maintenance and cannot update the path information promptly to adapt the environment changes. Similarly, the delivery ratio of the ISRO_PG decreases with the increase of the faillure nodes due to the less calculation.

Fig. 16 also supports the superiority of the MASM to the AODV, GA, SAW and ISRO_PG in the same environment, the performance remains high against the increas of the number of the nodes. While the number of nodes is small, the performence of these five algorithms are not huge different. Once the number of nodes exceeds 80 , the performance considerably and suddenly deteriorates in the GA. And the AODV and SAW can maintain the mean degree of the satisfaction even while there are 120 nodes. However, the delivery ratio in the MASM maintains a steady state with the increase of the nodes number than the AODV, SAW and ISPR_PG. Since the MASM is capable of finding a good solution as biological systems do, data is allocated to the other path while CP is busy or unable to work. This proposal improves network load balancing capacity and keeps the system stable.

\section{Conclusion}

In this paper we introduced a new heterogeneous IoT model which integrates the PLC and WiFi adaptively. This model is named MASM and based on the 3D Markov queuing model according to the user's priority and inspired method for multipath selection according to the adaptive response. The propsed algorithm has two steps. In the first step, data is divided into two priorities by the deadline delay of data. Then, we can obtain the mean transmission delay time of the different priorities according to the 3D Markov chain. The accuracy of evaluation results are analyzed by comparing the mathematical and simulation results. The aim of the second step is to select an optimal path for data. In this paper, we discuss the mathematical formulation of the MASM which is inspired by biology and only briefly outlined the path setup phase. Then, we map the results from the first step to the attractor, which is the important step in the MASM. Finally, the validity of the simulation method and thesystem stability are 
verified in the different scenarios by the simulation. Furthermore, the investigations of delivery ratios based different number nodes, compared with the AODV, GA, SAW and ISRO_PG, discussed in the heterogeneous IoT environment. The simulation results show the MASM has greatly improved network load balancing capacity and keeps the system stable.

\section{References}

[1] S. A. Alharthi, P. Johnson, M. A. Alharthi, "IoT architecture and routing for MV and LV smart grid,” in Proc. of 2017 Saudi Arabia Smart Grid (SASG), pp. 1-6, 2017. Article (CrossRef Link)

[2] Andreas Abart, Markus Flohberger, Reinhard Hirtler, "Assessment of electromagnetic fieldexposure in residential buildings caused by smart metering systems using PLC," CIRED-Open Access Proceedings Journal, vol. 1, no. 10, pp. 526-530, 2017. Article (CrossRef Link)

[3] Ala Al-Fuqaha, M. Guizani, M. Mohammadi, et al., "Internet of Things: A Survey on Enabling Technologies, Protocols, and Applications," IEEE Communication Surveys Tutorials, vol. 17, No. 4, pp. 2347-2376, 2015. Article (CrossRef Link)

[4] K. Ashton, “That 'internet of things' thing,” RFID Journal, vol. 2009, no. 22, pp. 97-114, 2009. Article (CrossRef Link)

[5] D. Lopez-Perez, M. Ding, H. Claussen, et al., "Toward 1Gbps/UE in cellular systems: Understanding ultra-dense small cell deployments," IEEE Communications Surveys \& Tutorials, vol. 17, no. 4, pp. 2078-2101, $4^{\text {th }}$ quarter 2015. Article (CrossRef Link)

[6] S. Kolipaka, B. N. Bhandari, and A. Dey, "Joint Admission Control and vertical handoff between WLAN and WIMAX in wireless mesh networks for QoS," in Proc. of 2016 IEEE International Conference on Engineering and Technology, pp. 1018-1023, 2016. Article (CrossRef Link)

[7] A. Gehlot, and A. Rajavat, "Handoff between WiMAX and WiFi wireless networks," in Proc. of 2016 Sympo- sium on Colossal Data Analysis and Networking, pp. 1-5, 2016.

Article (CrossRef Link)

[8] A. Blaise, E. Mohammed, and B. Yahya, et al., "HaVe-2W3G: a vertical handoff solution between WLAN, WiMAX and 3G networks," in Proc. of International Wireless Communications and Mobile Com- puting Conference, pp. 101-106, 2011. Article (CrossRef Link)

[9] H. Wu, X. M. Wen, Z. M. Lu, and Q. Pan, "Mobile data offloading under attractor selection in heterogeneous networks," in Proc. of 2017 International Symposium on Wireless Communication Systems (ISWCS), pp. 164-169, 2017. Article (CrossRef Link)

[10] H. Wu, X. M. Wen, Z. M. Lu, and Q. Pan, "Multi-access Selection with Attractor Selection Algorithm in Heterogeneous Network," in Proc. of 2015 IEEE/CIC International Conference on Communi- cations in China (ICCC), pp. 1-5, 2015. Article (CrossRef Link)

[11] F. Li, Y. Han, and C. Jin, "Practical access control for sensor networks in the context of the Internet of Things," Comput. Commun., vols. 89-90, pp. 154-164, Sep. 2016. Article (CrossRef Link)

[12] T. Hassan, S. Aslam, and J. W. Jang, "Fully Automated Multi-Resolution Channels and Multithreaded Spectrum Allocation Protocol for IoT Based Sensor Nets," IEEE Access, vol. 6, pp. 22545-22556, Mar. 2018. Article (CrossRef Link)

[13] S. Kunarak, and R. Sulessathira, "predictive RSS with fuzzy logic based vertical handoff algorithm in heterogeneous wireless networks," in Proc. of IEEE International Conference on Advanced Technologies for Communications, pp. 189-194, 2010. Article (CrossRef Link)

[14] J. Ying, K. Pahlavan, and X. Li, "Precision of RSS-based indoor geolocation in IoT applications," in Proc. of IEEE Annual International Symposium on Personal, Indoor, and Mobile Radio Communications, pp. 1-5, 2017. Article (CrossRef Link)

[15] K. Shanmugam, "A novel candidate network selection based handover management with fuzzy logic in heterogeneous wireless networks," in Proc. of IEEE International Conference on Advanced Comput- ing and Communication Systems, pp. 1-6, 2017. Article (CrossRef Link)

[16] Release 10. http://www.3gpp.org/specifications/releases/70-release-10 
[17] C. Chi, X. Cai and R. Hao, et al., “Modeling and analysis of handover algorithms,” IEEE GLOBECOM, pp. 4473-4477, Nov. 2007. Article (CrossRef Link)

[18] D. Ma and M. Ma, “A QoS oriented vertical handoff scheme for WiMAX/WLAN overlay networks,” IEEE Trans. Parallel Distrib. Syst., vol. 23, no. 4, pp. 598-606, 2012. Article (CrossRef Link)

[19] S. Lee, K, Kim, et al., "Vertical handoff decision algorithms for providing optimized performance in heterogeneous wireless networks,” IEEE Trans. Veh. Technol, vol. 58, no. 2, pp. 865-881, 2009. Article (CrossRef Link)

[20] R. Trestian, O. Ormond and G. Muntean, "Game theory-based network selection: Solutions and challenges,” IEEE Commun. Surveys Tutorials, vol. 14, no. 4, pp. 1212-1231, 2012. Article (CrossRef Link)

[21] J. Hou and D. C. O'Brien, "vertical handover-decision-making algorithm using fuzzy logic for the integrated Radio-and-OW system,” IEEE Trans. Wireless Commun., vol. 5, no. 1, pp. 176-185, 2006. Article (CrossRef Link)

[22] T. Taleb, A. Ksentini, “VECOS: a vehicular connection steering protocol,” IEEE Trans. Veh. Tech., vol. 64, no. 3, pp. 1171-1187, Mar.2015. Article (CrossRef Link)

[23] L. Chen, H. Li, "An MDP based vertical handoff decision algorithm for heterogeneous wireless networks," in Proc. of IEEE Wireless Communications and Networking Conference, April, 2016. Article (CrossRef Link)

[24] M. R. Palattella, et al., "Standardized protocol stack for the Internet of (important) things," IEEE Commun. Surveys Tuts., vol. 15, no. 3, pp. 1389-1406, 2013. Article (CrossRef Link)

[25] J. W. Hui and D. E. Culler, “Connecting low power and lossy network to the Internet," IEEE Commun. Mag., vol. 49, no. 4, pp. 96-101, 2011. Article (CrossRef Link)

[26] M. Segal, “A multiserver system with preemptive priorities,” Operations Research, vol. 18, no. 2, pp. 193-323, 1970. Article (CrossRef Link)

[27] N. TianandZ. G. Zhang, Vacation Queuing Models: Theory and Applications, Springer, New York, NY, USA, 2006. Article (CrossRef Link)

[28] N. Perel and U. Yechiali, “Queues with slow servers and impatient customers,” European Journal of Operational Research, vol. 201, no. 1, pp. 247-258, 2010. Article (CrossRef Link)

[29] U. Yechiali and P. Naor, “Queuing Problems with Heterogeneous Arrivals and Service,” Informs, vol. 19, no. 3, pp.559-841, 1971. Article (CrossRef Link)

[30] F. Mehmeti and T. Spyropoulos, "Performance Analysis of Mobile Data Offloading in Heterogeneous Networks,” IEEE Transactions on Mobile Computing, vol. 16, no. 2, pp.482-497, 2017. Article (CrossRef Link)

[31] K. Leibnitz, N. Wakamiya and M. Murata, "Biologically-inspired self -adaptive multi-path routing in overlay networks," Communications of the ACM, Special Issue on Self-Managed Systems and Services, vol. 49, no. 3, pp. 62-67, 2006. Article (CrossRef Link)

[32] K. Leibnitz, and M. Murata, “Attractor selection and perturbation for robust networks in fluctuating environments,” IEEE Network, Special Issue on Biologically Inspired Networking, vol. 24, no. 3, pp. 14-18, 2010. Article (CrossRef Link)

[33] S. Kajioka, N. Wakamiya, K. Leibnitz, and M. Murata, "Autonomous and adaptive resource allocation among multiple nodes and multiple application in heterogeneous wireless networks," Journal of Computer and System Sciences, vol. 78, no. 6, pp. 1673-1685, 2012. Article (CrossRef Link)

[34] H. Yu, Y. Ma, J. Yu, "Network selection algorithm for multiservice Multimode terminals in heterogeneous wireless networks,” IEEE Access, vol. 7, pp.46240-46260, 2019. Article (CrossRef Link)

[35] J. Zhao, "Research on Multi-Resources Optimization Strategies in Heterogeneous Wireless Networks,” Beijing University of Posts and Telecommunications, pp. 40-45, 2015. Article (CrossRef Link) 
[36] T. Zhong, T. Peng, and Y. Zhang, "A game theory scheme combined with the interference region in cellular D2D heterogeneous networks," in Proc. of IEEE/CIC International Conference on Communications in China (ICCC Workshops), pp. 167-171, 2018.

Article (CrossRef Link)

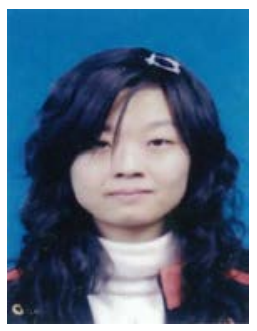

Wu Huan received his M. S. degrees from Kunming University of Science and Technology, Kunming, China, in 2010. She has worked as a lecturer in School of information engineering at West Anhui University from 2010 to 2012. She is currently a Ph. D. candidate at Beijing University of Post and Telecommunications, Beijing, China. Her current research interests are in the area of resource allocation in heterogeneous networks, PLC, network selection policy as well as the application of optimization theories in it.

Email:wuhuan279@gmail.com.

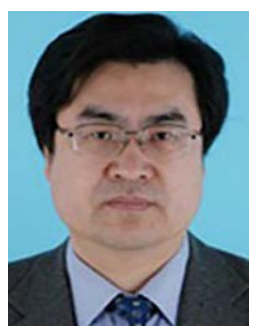

Xiangming Wen is the director of Beijing Key Laboratory of Network System Architecture and Convergence, where he has managed several projects related to open wireless networking. He is also the vice president of Beijing University of Posts and Telecommunications. He received both his M.S. and Ph.D. degrees in information and communication engineering from Beijing University of Posts and Telecommunications. His current research interests focus on radio resource and mobility management, software defined wireless networks, and broadband multimedia transmission technology.

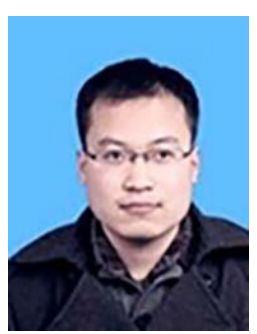

Zhaoming Lu received the Ph.D. in Beijing University of Posts and Telecommunications in 2012. He joined the School of Information and Communication Engineering in Beijing University of Posts and Telecommunications in 2012. His research includes Open Wireless Networks, QoE management in wireless networks, software defined wireless networks, cross-layer design for mobile video applications.

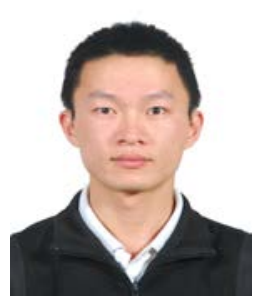

Nie Yao received his M. S. degrees from Kunming University of Science and Technology, Kunming, China, in 2009 and Ph. D. degrees from Beijing University of Post and Telecommunications, Beijing, China, in 2018, respectively. He joined the School of Information and Communication Engineering in West Anhui University in 2009. His current research interests are in the areas of wireless communications and networks, with an emphasis on polarization information processing and broadband multimedia transmission technology etc. Email: buptnieyao@gmail.com. 